\title{
Die neue Macht des Europäischen Parlaments nach ,Lissabon` im Bereich der gemeinsamen Handelspolitik
}

\author{
Elmar Brok*
}

Seit dem 1. Dezember 2009 macht ein Brüsseler Akteur mehr öffentliche Schlagzeilen als je zuvor: Das Europäische Parlament vertritt mit neuem Machtbewusstsein seine Positionen gegenüber Kommission, Rat und Mitgliedstaaten. Die Ablehnung des SWIFT-Abkommens ${ }^{1}$ durch das Europäische Parlament war sicherlich das prominenteste Beispiel der letzten Wochen. Dieses neue Selbstbewusstsein hat seine Gründe: Das Europäische Parlament gewinnt durch den Lissabon-Vertrag entscheidend an Bedeutung. Nicht umsonst wird der LissabonVertrag als „Vertrag der Parlamente“2 bezeichnet.

Ein konkretes Beispiel, an dem sich diese erweiterten Befugnisse und die neue ,Schlagkraft' des Europäischen Parlaments besonders herauskristallisieren, bietet ein Schlüsselbereich europäischer Politik: In der gemeinsamen Handelspolitik (GHP) der Europäischen Union gewinnen das Europäische Parlament und der Ausschuss für internationalen Handel des Europäischen Parlaments (INTA) an erheblicher Bedeutung. Bisher waren sie eher Randfiguren in der komplexen Struktur internationaler Handelspolitik. Auf europäischer Ebene agierten die Protagonisten Rat und Kommission. Da aber Teile der GHP, wie etwa ausländische Direktinvestitionen, nicht unter die ausschließliche gemeinschaftliche Zuständigkeit der Europäischen Union fielen, existierte hier parallel zu den EU-Regelungen ein wirres Geflecht aus bilateralen Übereinkommen der einzelnen Mitgliedstaaten mit Drittstaaten. Der Lissabon-Vertrag erweitert die Kompetenzen der Europäischen Union im Bereich der GHP mit dem Ziel, eine größere Einheitlichkeit europäischer Handelspolitik und somit eine größere Durchsetzungskraft europäischer Interessen auf globaler Ebene zu erreichen. Im Zuge dieser Reform bekommt das Europäische Parlament ebenfalls weitreichende neue Kompetenzen, die es von einem Nebenakteur zu einer Hauptfigur machen und zu einer ganz neuen Dimension und Demokratisierung der Handelspolitik führen könnten. Diese Neuerungen müssen im Kontext der allgemein erweiterten Kompetenzen des Europäischen Parlaments gesehen werden und der dahinterstehenden Motivation des Europäischen Konvents, die Europäische Union insgesamt demokratischer und transparenter zu machen.

Bevor auf die Details eingegangen und die neuen Regeln aufgezeigt und analysiert werden, lohnt sich ein Blick auf die Bedeutung der europäischen Handelspolitik im Allgemeinen und die durch den Lissabon-Vertrag neu hinzugekommenen Kompetenzen der Europäischen Union in der GHP, um vor diesem Hintergrund die Bedeutsamkeit und die Folgen der neuen Kompetenzen des Europäischen Parlaments und des INTA zu verstehen. Diese neuen

* Elmar Brok, MdEP, außenpolitischer Sprecher der „Fraktion der Europäischen Volkspartei“ im Europäischen Parlament, Vorsitzender der interparlamentarischen Delegation zum US-Kongress und des Transatlantic Legislators' Dialogue.

1 Europäische Kommission: Vorschlag für einen Beschluss des Rates über den Abschluss des Abkommens zwischen der Europäischen Union und den Vereinigten Staaten von Amerika über die Verarbeitung von Zahlungsverkehrsdaten und deren Übermittlung aus der Europäischen Union an die Vereinigten Staaten für die Zwecke des Programms zum Aufspüren der Finanzierung des Terrorismus, KOM (2009) 703. Das Kürzel ,SWIFT steht für „Society for Worldwide Interbank Financial Telecommunication”.

2 Elmar Brok/Martin Selmayr: Der, Vertrag der Parlamente' als Gefahr für die Demokratie? Zu den offensichtlich unbegründeten Verfassungsklagen gegen den Vertrag von Lissabon, in: integration 3/2008, S. 217-234. 
Kompetenzen werden einerseits in ihrer Konsequenz auf die interne Arbeit des Europäischen Parlaments analysiert. Woraus bestehen diese neuen Kompetenzen und welche Auswirkungen haben sie auf die Zusammenarbeit des INTA mit den anderen außenpolitischen Ausschüssen wie dem AFET (Ausschuss für Auswärtige Angelegenheiten) oder dem DEVE (Ausschuss für Entwicklung)? Welche Motivationen und Hintergründe waren für die nun weitreichenden Befugnisse des INTA ausschlaggebend und wie sind diese im Gesamtkontext der Stärkung des Europäischen Parlaments durch den Lissabon-Vertrag zu verstehen? Andererseits wird auf die externen Auswirkungen der neuen parlamentarischen Befugnisse in der GHP eingegangen: Was bedeutet eine Demokratisierung und Kontrolle der internationalen Handelspolitik? Wie wirkt sich diese auf das interinstitutionelle Gleichgewicht aus? Abschließend werden einige Empfehlungen für die Zukunft ausgesprochen.

\section{Wirtschaftlicher Riese und politischer Zwerg? Handelspolitik als Schlüsselbereich eu- ropäischer Außenpolitik}

Oftmals als außenpolitischer Zwerg beschimpft, ist die Europäische Union als wirtschaftlicher Riese schon lange weltweit akzeptiert. Die Zahlen sprechen für sich. Die Europäische Union repräsentiert heute mit ihren 27 Mitgliedstaaten circa 491 Millionen Menschen. Die Europäische Union steht mit ihren Erlösen aus Binnenmarkt und internationalem Handel für ein Drittel des Wohlstandes der Weltwirtschaft mit circa 31 Prozent des weltweiten Bruttoinlandsprodukts (BIP) (im Vergleich: USA 25,5 Prozent, Japan 8,1 Prozent, China 6,1 Prozent). ${ }^{3}$ Allein Deutschland exportierte im Januar 2010 Waren im Wert von 63,7 Milliarden Euro innerhalb und außerhalb der Europäischen Union. ${ }^{4}$

Neben dem innereuropäischen Handel im Rahmen des Binnenmarkts hat in den letzten Jahrzehnten auch der Außenhandel der Europäischen Union an Gewicht gewonnen. Wie Godelieve Quisthoudt-Rowohl in ihrem Beitrag ,Alle Macht in Brüssel: Die europäische Außenhandelspolitik“ richtig feststellt, hat die Bedeutung des Außenhandels in den letzten Jahren durch ,den technologischen Fortschritt, der für eine Verringerung der Kommunikations- und Transportkosten gesorgt hat" ${ }^{5}$, zugenommen. Weitere Gründe für die gestiegene Relevanz der Außenhandelspolitik sieht Quisthoudt-Rowohl in politischen Prozessen und Entscheidungen wie dem schrittweisen Abbau von Handelshemmnissen auf multilateraler Ebene, Chinas wirtschaftlicher Öffnung seit 1978 und dem Fall des Eisernen Vorhangs. Parallel seien an den multilateralen Handelsverhandlungen immer mehr und immer ehrgeizigere aufstrebende Wirtschaftsmächte beteiligt. Das Gewicht und die Wichtigkeit, die die Handelspolitik für den Wohlstand und die wirtschaftliche Schlagkraft der Europäischen Union hat, werde deutlich, wenn man sich vor Augen führt, dass mittlerweile circa jeder vierte Arbeitsplatz vom Export abhängt. ${ }^{6}$ Die Europäische Union ist in der Tat der größte Exporteur von Waren und Dienstleistungen. So beträgt der Anteil der ,EU-27` am weltweiten Güterhandel 17,8 Prozent (USA 14,0 Prozent, China 10,4 Prozent, $\mathrm{ASEAN}^{7}$ 7,8 Prozent, Lateinamerika 7,1 Prozent). Bei

3 United Nations Conference on Trade and Development: UNCTAD Handbook of Statistics 2008, New York/ Genf, S. 388.

4 Eurostat: Handelsbilanzüberschuss der Eurozone bei 2,6 Mrd. Euro. Defizit von 6,0 Mrd. Euro für die EU27, Pressemitteilung 51/2010, 16.04.2010.

5 Godelieve Quisthoudt-Rowohl: Alle Macht in Brüssel: Die europäische Außenhandelspolitik, in: Kurt J. Lauk (Hrsg.): Europa von innen gesehen: Europa jenseits der Bürger? Die EU nach dem Vertrag von Lissabon, Stuttgart 2009, S. 144-176, S. 145.

6 Ebenda.

7 „Association of Southeast Asian Nations“. 
Dienstleistungen liegt die Europäische Union sogar mit 26,1 Prozent an der Spitze und überragt dabei bei Weitem die USA mit 17,3 Prozent, die an zweiter Stelle folgen. ${ }^{8}$

Als größte Exportmacht ist die Europäische Union auch für eine beträchtliche Zahl von Drittstaaten der wichtigste Handelspartner. Monatlich fließen Massen an Importen in die Europäische Union. Allein im Februar 2010 wurden Waren im Wert von 102,3 Milliarden Euro in die ,EU-27 ‘ importiert. ${ }^{9}$ So nahm der Handel der ,EU-27` mit wichtigen Partnerländern $\mathrm{zu}$ - bei den Ausfuhren nach China im Januar 2010 sogar um 49 Prozent im Vergleich zum Vorjahr. ${ }^{10}$ Auch am Beispiel USA sieht man deutlich die Dimensionen und die Bedeutsamkeit der Handelsbeziehungen mit Drittstaaten für unsere heimische Wirtschaft: Die transatlantische Wirtschaft generiert pro Jahr knapp 60 Billiarden Euro. Somit hängen insgesamt fast 14 Millionen Arbeitsplätze von der transatlantischen Wirtschaft ab. ${ }^{11}$ Der 2007 gegründete Transatlantische Wirtschaftsrat visiert sogar für 2015 das Ziel der Schaffung eines echten transatlantischen Markts an. Gelänge dies, wäre es ein riesiger Erfolg. Das BIP könnte in der Europäischen Union bis 2018 um 0,7 Prozent pro Jahr und in den USA bis 2018 um 0,3 Prozent pro Jahr zusätzlich steigen!12 Global erwirtschaften Europäer und Nordamerikaner derzeit bereits zusammen 60 Prozent des globalen BIP.

Die Europäische Union ist also unumstritten ein ,wirtschaftlicher Riese‘. Das heißt aber nicht im Umkehrschluss, wie oft suggeriert wird, dass sie ein ,politischer Zwerg' ist. Das Bild ,wirtschaftlicher Riese versus politischer Zwerg' geht nicht auf. Dies wird deutlich, wenn man sich bewusst macht, dass gerade der Bereich des Außenhandels auch ein wichtiges Instrument der Außenpolitik ist, welches neben den wirtschaftlichen Interessen eine Art ,Hebelwirkung f für die Durchsetzung anderer außenpolitischer Ziele entwickeln kann. Dies wird in der Öffentlichkeit oftmals nicht beachtet. All zu schnell setzt man Außenpolitik mit Sicherheitspolitik gleich und vergisst dabei den wichtigen Bereich des Außenhandels. Der neue EU-Außenhandelskommissar Karel De Gucht definierte bei einer Anhörung im Europäischen Parlament am 12. Januar 2010 die Ziele und den Zweck gemeinsamer EU-Handelspolitik wie folgt: „Freier Handel muss ein Mittel sein, um Wohlstand, Stabilität und Entwicklung zu fördern." ${ }^{13}$ Und genau darum geht es - internationale Handelspolitik ist mehr als das Importieren und das Exportieren von Waren. Sie muss immer im Gesamtkontext außenpolitischer Beziehungen gesehen werden. Sie ist auch politisches, diplomatisches Instrument. Der Abschluss eines Handelsabkommens kann und wird in den meisten Fällen an andere Bedingungen geknüpft, konditionalisiert. Handel ist eine Dimension, ein Teil der außenpolitischen ,tool box ' der Europäischen Union, die neben dem Waren- und Dienstleistungsaustausch über unsere Grenzen hinweg unsere Werte in der Welt verbreitet und unsere Interessen durchsetzt. Die Einhaltung von Menschenrechten und Handel sind kaum voneinander zu trennen. Genauso geht es im Bereich der Handelspolitik auch immer um Entwicklungspolitik und um Sicherheitspolitik - man denke an Iran und das mögliche Druckmittel wirtschaftlicher Sanktionen.

8 UNCTAD Handbook of Statistics 2008, S. 388.

9 Eurostat: Handelsbilanzüberschuss, 2010.

10 Ebenda.

11 Daniel S. Hamilton/Joseph P. Quinlan: The Transatlantic Economy 2010. Annual Survey of Jobs, trade and Investment between the United States and Europe, Washington D.C. 2010, S. V.

12 Laut einer Studie von Koen G. Berden/Joseph Francois/Martin Thelle/Paul Wymenga/Saara Tamminen: NonTariff Measures in Trade and Investment - An Economic Analysis, Rotterdam 2009, S. 70.

13 Rede von Karel de Gucht anlässlich seiner Anhörung im INTA am 12. Januar 2010. Vgl. Europäisches Parlament, Ausschuss für internationalen Handel: Hearing of Karel De Gucht Commissioner-Designate Trade, 12.01.2010, inoffizielle Niederschrift, S. 6, abrufbar unter: http://www.europarl.europa.eu/hearings/static/commissioners/cre/de_gucht.pdf (letzter Zugriff: 14.05.2010). 
Durch Handel schaffen wir es Zugang zu anderen Märkten und - das ist das Entscheidende - zu anderen Gesellschaften zu bekommen. China, welches Jahrzehnte lang verschlossen war, hat nach und nach seinen Markt geöffnet. Dadurch bekommen wir mehr und tiefere Einblicke und auch mehr Einfluss und Möglichkeiten, die Verhältnisse im Inneren zu ändern und Druck auf die chinesische Regierung auszuüben. Außenhandelspolitik ist also ein Instrument der sogenannten ,soft power', welches andere diplomatische Mittel und auBenpolitische Instrumente der Europäischen Union (wie zum Beispiel das Stabilitäts- oder das Krisenmanagementinstrument) ergänzen und somit den Griff zu militärischen Mitteln verhindern kann. Ganz konkret werden oftmals Handelsabkommen konditionalisiert, zum Beispiel an die Einhaltung von menschenwürdigen Bedingungen am Arbeitsplatz geknüpft.

Und nicht nur das: Außenhandelspolitik ist auch eng gekoppelt mit innenpolitischen, wirtschaftlichen Maßnahmen. Wieder macht es De Gucht deutlich: „Ich werde dafür eintreten, dass die Handelspolitik bei der Verwirklichung der neuen Vision für 2020 voll zum Tragen kommt, indem die politischen Maßnahmen im Innern mit der Stärkung der externen Wettbewerbsfähigkeit der Europäischen Union gekoppelt werden."14 Denn Handel spornt auch zur Innovation und Kreativität, zur Effizienzsteigerung und Qualität unserer Unternehmen an, die sich nur dadurch im globalen Wettbewerb durchsetzen können. Wir sehen also die weittragende Bedeutung der Handelspolitik. Sie ist für den Exportweltmeister Europäische Union nicht nur das Standbein für Wohlstand und Wohlfahrt innerhalb der Europäischen Union, sondern auch ein Instrument der Außenpolitik, das auch außerhalb der Europäischen Union zu mehr Frieden, Demokratie und Wohlstand seinen Beitrag leistet und unsere in der Grundrechtecharta der Europäischen Union und in Art. 21 EUV $^{15}$ definierten Grundwerte in der Welt verbreitet.

\section{Gemeinsame Außenhandelspolitik als logische Folgerung des Binnenmarktes}

Schon Tucholsky erkannte in seiner Weltbühne 1931: „Was die Weltwirtschaft angeht, so ist sie verflochten. "16 Dies gilt umso mehr für die europäische Wirtschaft, die durch den Binnenmarkt eng miteinander verbunden ist. Da die Europäische Union einen gemeinsamen Markt teilt und eine gemeinsame Grenze hat, ist die logische Folgerung auch eine GHP. Bisher war es laut Art. $131 \mathrm{EGV}^{17}$ das Hauptziel der GHP ,im gemeinsamen Interesse zur harmonischen Entwicklung des Welthandels, zur schrittweisen Beseitigung der Beschränkungen im internationalen Handelsverkehr und zum Abbau der Zollschranken beizutragen. “ Zu diesem Zweck hatte die GHP bisher zwei Hauptaufgaben: Zum einen geht es in der sogenannten vertraglichen Handelspolitik darum, bei internationalen Verhandlungen und Drittstaaten gegenüber als einheitlicher, starker verhandlungsfähiger Partner nach außen hin aufzutreten und Handelsabkommen mit Drittstaaten zu verhandeln, zu ratifizieren und zu implementieren. Zum anderen ist es aber im Rahmen der sogenannten internen/autonomen

14 Antworten von Karel de Gucht auf die Fragen des INTA für die Anhörung im Europäischen Parlament am 4. Januar 2010. Vgl. Europäisches Parlament, Ausschuss für internationalen Handel: Mitteilung an die Mitglieder des INTA-Ausschusses, PE431.166v01-00, S. 9, abrufbar unter: http://www.europarl.europa.eu/meetdocs/ 2009_2014/documents/inta/dv/800/800569/800569de.pdf (letzter Zugriff: 11.05.2010).

15 Die Abkürzung ,EUV ‘ bezieht sich auf die konsolidierte Fassung des Vertrags über die Europäische Union in der Fassung des Lissabon-Vertrags, in: Amtsblatt der EU, Nr. C 83 vom 30. März 2010, S. 13-45.

16 Kurt Tucholsky (Kaspar Hauser): Kurzer Abriss der Nationalökonomie, in: Die Weltbühne, 15.09.1931, S. 37, wiederabgedruckt in: Mary Gerold-Tucholsky/Fritz J. Raddatz (Hrsg.): Kurt Tucholsky. Gesammelte Werke 3. 1929-1935, Frankfurt am Main 2005, S. 939-941, hier S. 939.

17 Sofern nichts anderes vermerkt ist, bezieht sich die Abkürzung ,EGV‘ auf die konsolidierte Fassung des Vertrags zur Gründung der Europäischen Gemeinschaft in der Fassung des Vertrags von Nizza, in: Amtsblatt der EU, Nr. C 321 E vom 29. Dezember 2006, S. 37-180. 
Handelspolitik auch Aufgabe der GHP, handelspolitische Instrumente wie Zölle, gemeinsame Einfuhrregelungen, Anti-Dumpingmaßnahmen, subventionierte Importe, mengenmäBige Beschränkungen, Exporterstattungen und die Gewährung gegenseitiger Handelspräferenzen anzuwenden. ${ }^{18}$ Außerdem müssen im Rahmen dieser Politik interne Regeln an internationale Vereinbarungen angepasst und vereinheitlicht werden. Ein wichtiges Stichwort in diesem Zusammenhang ist die Harmonisierung von verschiedenen Rechtsvorschriften der Nationalstaaten, sodass Wettbewerbsverzerrungen entgegengetreten werden kann und die Europäische Union durch ihre Einheitlichkeit eine höhere Handlungsfähigkeit und Durchsetzungskraft erreicht. So verteidigt die Europäische Kommission zum Beispiel die Interessen der EU-Mitgliedstaaten auf globaler Ebene und achtet zugleich auf die Einhaltung der Regeln, sodass einheimischen Produzenten keine Nachteile durch die Einfuhr von Gütern entstehen. Ein prominentes Beispiel hierfür ist die sogenannte ,Antidumpingverordnung' $\cdot 19$

Hauptakteure innerhalb der GHP waren bisher die Europäische Kommission und der Rat. Während die Kommission das Vorschlags- und Verhandlungsmonopol besaß und die Europäische Union bei internationalen Verhandlungen vertrat, wurden Abkommen vom Rat geschlossen. ${ }^{20}$ Das Europäische Parlament und der INTA spielten dabei nur eine Nebenrolle. Den EG-Verträgen zufolge war eine Anhörung des Europäischen Parlaments bei GHP-Maßnahmen und bei dem Abschluss von Handelsabkommen bisher nicht vorgesehen. In Art. 133 Abs. 2 EGV heißt es in Bezug auf die GHP: „Die Kommission unterbreitet dem Rat Vorschläge für die Durchführung der gemeinsamen Handelspolitik.“ Maßnahmen im Rahmen der GHP und der Abschluss von Handelsabkommen wurden allein von der Kommission ausgehandelt und allein vom Rat beschlossen. Eine Anhörung des Europäischen Parlaments in Bezug auf den Abschluss von Handelsabkommen wurde sogar explizit ausgeschlossen. ${ }^{21}$ Das Parlament war bisher nur dann am Abschluss solcher Abkommen beteiligt, wenn der betreffende Vorschlag mehr als eine Rechtsgrundlage hatte..$^{22}$

In der Praxis sah das allerdings etwas anders aus: In der Rahmenvereinbarung über die Beziehungen zwischen dem Europäischen Parlament und der Europäischen Kommission war nämlich Folgendes festgelegt: ,In Bezug auf internationale Abkommen, darunter auch Handelsabkommen, unterrichtet die Kommission das Parlament frühzeitig und eindeutig sowohl während der Phase der Vorbereitung der Abkommen als auch während des Verlaufs und des Abschlusses internationaler Verhandlungen. [...] Die Unterrichtung des Parlaments [...] erfolgt so rechtzeitig, dass es erforderlichenfalls seinen Standpunkt zum Ausdruck bringen kann und die Kommission den Standpunkten des Parlaments im Rahmen des Möglichen Rechnung tragen kann. ${ }^{23}$

18 Vgl. Benjamin Fairbrother/Godelieve Quisthoudt-Rowohl: Europäische Handelspolitik: Von Rom bis Lissabon, in: Konrad Adenauerstiftung (Hrsg.): Analysen und Argumente, Ausgabe 73, Januar 2010, S. 7.

19 Verordnung (EG) Nr. 384/96 des Rates vom 22. Dezember 1995 über den Schutz gegen gedumpte Einfuhren aus nicht zur Europäischen Gemeinschaft gehörenden Ländern, in: Amtsblatt der EU, Nr. L 56 vom 6. März 1996, S. 1-20.

20 Bei den sogenannten, gemischten Abkommen " mussten neben dem Rat auch die einzelnen Mitgliedstaaten ihre Zustimmung geben.

21 Art. 300 Abs. 3 EGV.

22 Beispiele sind Mitentscheidungen bei Rechtsakten, die auf der doppelten Rechtsgrundlage von Art. 133 und 95 EGV fußten, Zustimmungen zu Abkommen, die einen der Aspekte des Art. 300 Abs. 3 UAbs. 2 EGV beinhalteten, oder Ausnahmefälle, wenn der Rat das Parlament auf freiwilliger Grundlage anhörte.

23 Rahmenvereinbarung zwischen dem Europäischen Parlament und der Kommission. Beschluss des Europäischen Parlaments zu der Revision der Rahmenvereinbarung zwischen dem Europäischen Parlament und der Kommission (2005/2076(ACI)), in: Amtsblatt der EU, Nr. C 117 E vom 18. Mai 2006, S. 123-133, hier S. 128. 
Dies bedeutete praktisch, dass der INTA zwar Dokumente und Informationen im Zusammenhang mit der Verhandlung von Handelsabkommen bekam und der Handelskommissar zum Meinungsaustausch im INTA erschien. Dies fand aber auf einer informellen, nicht verbindlichen Ebene statt. In Wirklichkeit war das Europäische Parlament machtlos.

Selbst in einigen wenigen Sonderfällen, in denen das Europäische Parlament ein Zustimmungsrecht besaß, war dieses in der Praxis oftmals nicht mehr als eine leere Hülle. So hatte das Europäische Parlament zum Beispiel bei dem Abkommen zur Gründung der „World Trade Organization“ (WTO) ein Zustimmungsrecht. ${ }^{24}$ Dieses wurde aber zu einer Farce, da das Europäische Parlament unter enormem Zeitdruck stand und drei Monate nachdem es das 26.000 Seiten umfassende Abkommen erhalten hatte schon abstimmte. Eine wirklich kritische Prüfung durch das Europäische Parlament, das unter erheblichem Druck von Kommission und Rat stand, war hier mehr als zweifelhaft. 25

Trotz der im Vergleich zum Europäischen Parlament mächtigen Rolle der Kommission muss eines nochmals deutlich hervorgehoben werden: Nach den EG-Verträgen waren auch die Rechte der Kommission im Bereich der GHP lange Zeit insofern begrenzt, als dass im Vertrag von Maastricht laut Art. 133 Abs. 1 EGV sich die ausschließliche Zuständigkeit nicht auf alle Aspekte des Handels bezog, sondern nur auf „die Änderung von Zollsätzen, den Abschluss von Zoll- und Handelsabkommen, die Vereinheitlichung der Liberalisierungsmaßnahmen, die Ausfuhrpolitik und die handelspolitischen Schutzmaßnahmen, zum Beispiel im Fall von Dumping und Subventionen." Im Bereich des Handels mit Dienstleistungen, des geistigen Eigentums oder ausländischen Direktinvestitionen hatte die Europäische Union lange Zeit nicht die ausschließliche Zuständigkeit. ${ }^{26}$ Vielmehr lag hier oftmals eine sogenannte, gemischte Zuständigkeit ${ }^{‘ 27}$ vor. Durch den Vertrag von Nizza wurde hier allerdings ein erster Fortschritt erreicht. Art. 133 Abs. 5 EGV schloss nun explizit den Handel mit Dienstleistungen und die handelsbezogenen Aspekte des geistigen Eigentums in die GHP mit ein. Der Bereich der ausländischen Direktinvestitionen blieb hingegen weiterhin ausgeschlossen. ${ }^{28}$

\section{Neue Reichweite und Verzahnung der GHP durch den Lissabon-Vertrag}

Gegenüber den bisherigen Bestimmungen wird die GHP nun im Lissabon-Vertrag erheblich ausgebaut. Erstens wird die GHP nun in allen Bereichen der Handelspolitik eine gemeinschaftliche Handelspolitik. Der Vertrag dehnt die substanzielle Reichweite der GHP

24 Hier handelt es sich um einen Sonderfall. Das Europäische Parlament hatte ein Zustimmungsrecht, da es um die Schaffung eines besonderen institutionellen Rahmens ging. Vgl. Fairbrother/Quisthoudt-Rowohl: Europäische Handelspolitik, 2010, S. 10.

25 Ebenda. Vgl. auch Meinhard Hilf/Frank Schorkopf: Das Europäische Parlament in den Außenbeziehungen der Europäischen Union, in: Josef Drexl u.a. (Hrsg.): Europäische Demokratie, Baden-Baden 1999, S. 109-129, hier S. 112. Vgl. auch Ernst-Ulrich Petersmann: Welthandelsrecht als Freiheits- und Verfassungsordnung, in: Zeitschrift für ausländisches öffentliches Recht und Völkerrecht 65/2005, S. 543-585, hier S. 546.

26 Die Frage nach der Reichweite der handelspolitischen Kompetenzen der Europäischen Union war laut dem Vertrag von Maastricht unklar. Es war nicht klar, ob die Aufzählung von Art. 133 EGV in der Fassung des Vertrags von Maastricht erschöpfend gemeint war oder nur Beispiele auflisten sollte. Anfang der 1990er Jahre entschied der Europäische Gerichtshof, dass die Zuständigkeit für den Handel mit Dienstleistungen und handelsbezogenen Aspekten des geistigen Eigentums in die gemischte Zuständigkeit fiel, also die Zuständigkeit zwischen der Europäischen Union und den Mitgliedstaaten geteilt war. Vgl. Fairbrother/Quisthoudt-Rowohl: Europäische Handelspolitik, 2010, S. 8-9.

27 Bei gemischten Zuständigkeiten handelt es sich um geteilte Zuständigkeiten zwischen Europäischer Union und Mitgliedstaaten. Hier gilt nicht das Prinzip der qualifizierten Mehrheitsentscheidung im Rat, sondern jeder Mitgliedstaat muss zustimmen. Außerdem müssen zudem noch die nationalen Parlamente der Mitgliedstaaten zustimmen.

28 Vgl. Fairbrother/Quisthoudt-Rowohl: Europäische Handelspolitik, 2010, S. 8-9. 
auf alle Handelsbereiche aus. So ist in Art. 3 Abs. 1 Lit. e AEUV ${ }^{29}$ die Handelspolitik als ein Politikbereich aufgeführt, der in die ,ausschließliche Zuständigkeit“ der Europäischen Union fällt. Das war aber, wie beschrieben, in vielen Bereichen schon vorher so. Das Entscheidende ist vielmehr, dass in Art. 207 AEUV die Bereiche der Handelspolitik gegenüber dem ehemaligen Art. 133 EGV ausgeweitet werden. So fällt nun auch der Bereich der ausländischen Direktinvestitionen unter die ausschließliche Zuständigkeit der Europäischen Union.

Und dies ist entscheidend! Die Europäische Union hat in nunmehr allen Bereichen der Handelspolitik die ausschließliche Zuständigkeit. Die Ausdehnung auf ausländische Direktinvestitionen stellt einen großen Fortschritt dar. Zwar war die Europäische Union schon vorher für Aspekte der internationalen Marktöffnung für Investitionen zuständig, ${ }^{30}$ aber alle anderen Maßnahmen, die den Schutz bei Direktinvestitionen im Ausland garantieren sollten, waren Verhandlungssache der einzelnen Nationalstaaten. Folge war ein undurchschaubares Geflecht an bilateralen Vereinbarungen. Dies erwies sich in der Praxis oft als Nachteil: Wenn beispielsweise ein europäisches Unternehmen mit Sitzen in mehreren EU-Mitgliedstaaten in China investieren wollte, war es bisher nicht sicher, ob die Investitionen des in Deutschland ansässigen Unternehmens genau nach den gleichen Regeln geschützt wurden, wie die des in Frankreich ansässigen Tochterunternehmens.

Zweitens trägt der Lissabon-Vertrag der bereits beschriebenen Verzahnung von Außenhandelspolitik mit anderen Aspekten der Außenpolitik wie Entwicklungs-, Menschenrechtsund Sicherheitspolitik Rechnung, indem er die GHP in den größeren Rahmen europäischer Außenpolitik einbettet. Laut Art. 207 AEUV wird ,die gemeinsame Handelspolitik [...] im Rahmen der Grundsätze und Ziele des auswärtigen Handelns der Union gestaltet.“ Der bereits erwähnte Art. 131 EGV wird also erheblich ausgeweitet und die GHP über das Ziel des Abbaus von Handelshemmnissen hinaus in einem viel größeren, komplexeren Kontext dem breiten Feld des auswärtigen Handelns - definiert. Die erwähnten „Grundsätze und Ziele des auswärtigen Handelns“ sind in Art. 21 Abs. 1 EUV festgelegt. Es heißt hier: „Die Union lässt sich bei ihrem Handeln auf internationaler Ebene von den Grundsätzen leiten, die für ihre eigene Entstehung, Entwicklung und Erweiterung maßgebend waren und denen sie auch weltweit zu stärkerer Geltung verhelfen will: Demokratie, Rechtsstaatlichkeit, die universelle Gültigkeit und Unteilbarkeit der Menschenrechte und Grundfreiheiten, die Achtung der Menschenwürde, der Grundsatz der Gleichheit und der Grundsatz der Solidarität sowie die Achtung der Grundsätze der Charta der Vereinten Nationen und des Völkerrechts." Insbesondere wichtig in Bezug auf den Außenhandel ist neben der Stärkung der Grundwerte wie Demokratie, Rechtsstaatlichkeit und Menschenrechte das in Art. 21 Abs. 2 Lit. e EUV festgelegte Ziel ,die Integration aller Länder in die Weltwirtschaft zu fördern, unter anderem durch den schrittweisen Abbau internationaler Handelshemmnisse."

Eine weitere Neuerung besteht darin, dass in Art. 206 AEUV ausdrücklich der Abbau ,anderer Schranken“ erwähnt wird. Damit sind „,nichttarifäre Beschränkungen“ des internationalen Handels gemeint, die ,gegenüber den traditionellen Zöllen zugenommen“ haben ${ }^{31}$ und die, ebenso wie Zölle, dem Schutz der heimischen Produzenten dienen. Sie bestehen

29 Vertrag über die Arbeitsweise der Europäischen Union, in: Amtsblatt der EU, Nr. C 83 vom 30. März 2010, S. 47-199.

30 Zum Beispiel wurden dienstleistungsbezogene Investitionen im „General Agreement on Trade in Service“ (GATS) verhandelt.

31 Andreas Maurer/Bettina Rudloff: Handelspolitik und Internationale Abkommen, in: Julia Lieb/Nicolai von Ondarza/Torsten Schütz (Hrsg.): SWP-Webdossier, „Der Vertrag von Lissabon und seine Umsetzung“, Februar 2010. 
zum Beispiel in Verpackungsvorgaben, normtechnischen Vorgaben oder auch Ansprüchen an die Qualifikation von Dienstleistungserbringern.

Insgesamt sieht man, dass durch die Übertragung der ausschließlichen Zuständigkeit an die Europäische Union in nunmehr allen Bereichen der GHP und ihrer damit einhergehenden größeren substanziellen Reichweite sowie der Einbettung der GHP in den Kontext des auswärtigen Handelns ,eine Anpassung an die gewandelten Realitäten des internationalen Wirtschaftssystems“"32 erfolgte. Denn im europäischen Wirtschaftssystem, welches durch den Binnenmarkt unsere Wirtschaften und somit die Grundlage unseres Wohlstands und sozialen Friedens miteinander verbindet, muss man auch global nach außen gemeinsam und einheitlich auftreten. Nur so kann man die nötige Schlagkraft entwickeln und die Durchsetzungskraft unserer wirtschafts- und handelspolitischen Interessen garantieren. Ebenso war die Erkenntnis entscheidend, dass es in einer ,EU der $27^{\circ}$ immer schwieriger wurde, Einstimmigkeit in wichtigen handelspolitischen Fragen zu erzielen. Deshalb lag es nahe, Mehrheitsentscheidungen innerhalb der GHP auszuweiten. ${ }^{33}$ Die neuen Kompetenzen der Europäischen Union in der GHP symbolisieren diese Einsicht.

\section{Die neuen Befugnisse des Europäischen Parlaments in der GHP}

Der Lissabon-Vertrag sieht im Zuge der dargestellten erweiterten Kompetenzen der Europäischen Union in der GHP einen enormen Machtzuwachs des Europäischen Parlaments im Bereich der GHP vor. Dieser erscheint umso signifikanter, da die GHP insgesamt, wie dargestellt, aufgewertet wurde.

Das Europäische Parlament erhält nun Kontroll- und Mitentscheidungsbefugnisse, die es auf eine Ebene mit dem Ministerrat stellen. Praktisch obliegt seit dem 1. Dezember 2009 die gesamte Handelspolitik der Kozuständigkeit des Europäischen Parlaments - und hier vor allem seiner , vorbereitenden Hand': des erst 2004 gegründeten INTA.

Konkret gewinnt das Europäische Parlament folgende Befugnisse: Erstens dehnt der Lissabon-Vertrag das in ordentliches Gesetzgebungsverfahren umbenannte Mitentscheidungsverfahren in der Gesetzgebung auf die autonome Handelspolitik aus. In Art. 207 Abs. 2 AEUV heißt es: „Das Europäische Parlament und der Rat erlassen durch Verordnungen gemäß dem ordentlichen Gesetzgebungsverfahren die Maßnahmen, mit denen der Rahmen für die Umsetzung der gemeinsamen Handelspolitik bestimmt wird.“ Europäisches Parlament und Rat sind nun in diesem Bereich der GHP gleichberechtigte Mitgesetzgeber. Diese Gleichberechtigung gilt auch für Maßnahmen der wirtschaftlichen, finanziellen und technischen Zusammenarbeit zugunsten von Drittländern, die nicht zur Gruppe der Entwicklungsländer gehören. Dies bedeutet einen enormen Fortschritt! Konnte das Europäische Parlament bisher nur Stellungnahmen zur Gesetzgebung im Bereich der autonomen GHP abgeben, wird es nun Mitgesetzgeber und ist somit mitverantwortlich für alle Gesetze, die den Rahmen für die Umsetzung der GHP betreffen.

Zweitens gilt die neue Qualität der Parlamentsrechte insbesondere für das Feld der internationalen Verhandlungen. Das Europäische Parlament wird nun im Rahmen der vertraglichen Handelspolitik durch das Zustimmungsverfahren beteiligt. Es muss also alle Handelsabkommen ratifizieren. Außerdem bekommt es in der vertraglichen Handelspolitik neue Kontroll- und Informationsrechte. Der Vertrag sieht in Art. 207 Abs. 3 AEUV explizit vor, dass das Europäische Parlament in allen Phasen der Verhandlungen informiert und konsul-

32 Till Müller-Ibold: Gemeinsame Handelspolitik, in: Carl-Otto Lenz/Klaus-Dieter Borchardt (Hrsg.): EU-Verträge. Kommentar nach dem Vertrag von Lissabon, Köln 2010, S. 2205-2264, hier S. 2207.

33 Vgl. Fairbrother/Quisthoudt-Rowohl: Europäische Handelspolitik, 2010, S. 9. 
tiert werden muss, und zwar in derselben Weise wie der vom Ministerrat eingesetzte, seit 1958 ohne jede unmittelbar-demokratische Verankerung operierende sogenannte „133er Ausschuss “. ${ }^{34}$ Die Kommission ist nun also rechtlich verpflichtet, Informationen frühzeitig an das Europäische Parlament weiterzuleiten. Konnte das Europäische Parlament bisher durch das Rahmenabkommen zwischen Parlament und Kommission von 2005 zwar informell informiert und konsultiert werden, wird dieses nun rechtlich fixiert und somit verbindlich. Dementsprechend heißt es in der Entschließung des Europäischen Parlaments zu den Eckpunkten des neuen Rahmenabkommens zwischen Kommission und Parlament vom 9. Februar 2010, dass die Kommission das Europäische Parlament frühzeitig und detailliert über Entwürfe für Verhandlungsmandate und Änderungen dieser Mandate informieren soll. ${ }^{35}$ Deutlich sicherte Karel De Gucht anlässlich seiner Anhörung im Europäischen Parlament am 12. Januar $2010 \mathrm{zu}$, dass es ihm ein besonderes Anliegen sei, das Parlament ständig zu informieren und ihm die Gelegenheit zu geben, sich in allen Verhandlungsphasen zu äuBern. ${ }^{36}$

Das Europäische Parlament hat allerdings weiterhin nicht das Recht, das Verhandlungsmandat zu bestimmen. Es obliegt nach wie vor ausschließlich der Kommission, die Verhandlungen für ein Handelsabkommen zu führen. ${ }^{37}$ So bleibt eine Diskrepanz zwischen den Kompetenzen des Europäischen Parlaments bei EU-internen Bereichen der GHP (autonome Handelspolitik) und den externen Bereichen der GHP (vertragliche Handelspolitik) bestehen.

Die Dimension der neuen Befugnisse des Europäischen Parlaments im Gesetzgebungsverfahren wird deutlich, wenn man einen Blick zurück wirft. In der Vergangenheit waren das Europäische Parlament und der INTA, wie bereits erwähnt, eher eine Randfigur. In den meisten Fällen wurden sie nur konsultiert, hatten aber nichts zu sagen. So wurden in der vergangenen Legislaturperiode innerhalb von fünf Jahren gerade nur zwei Mitentscheidungsverfahren durchgeführt, ${ }^{38}$ die restlichen Verfahren waren hauptsächlich Konsultationsverfahren. ${ }^{39}$ Dieses wird sich nun aller Erwartung nach ändern. Dadurch dass die GHP im Allgemeinen eine viel größere Schlagkraft erhält und zudem die demokratische Kontrolle und Transparenz durch die erweiterten Befugnisse des Europäischen Parlaments gestärkt werden, erhält das Europäische Parlament nun eine ganz andere Relevanz und wird auch von außen völlig anders wahrgenommen. Dies merkt man allein an der Anzahl der Interessenvertreter, die nun in weitaus größerer Anzahl als bisher die Ausschusssitzungen des INTA aufmerksam verfolgen und den Kontakt zu seinen Mitgliedern suchen. Auf einmal ha-

34 Die informelle Bezeichnung ,133er Ausschuss“ rührt von der Tatsache, dass vor dem Lissabon-Vertrag dieses Gremium auf Basis des Art. 133 Abs. 3 EGV eingesetzt wurde. Im Lissabon-Vertrag ist der „133er Ausschuss“ nun in Art. 207 Abs. 3 AEUV als vom Rat bestellter „Sonderausschuss“ genannt.

35 Vgl. Maurer/Rohloff: Handelspolitik und Internationale Abkommen, 2010.

36 Europäisches Parlament, Ausschuss für internationalen Handel: Hearing of Karel De Gucht, 2010, S. 7, 10 und 30.

37 Art. 207 Abs. 3 und Art. 218 Abs. 2 AEUV.

38 Europäisches Parlament: Bericht des Ausschusses für internationalen Handel über den Vorschlag für eine Verordnung des Europäischen Parlaments und des Rates über Gemeinschaftsstatistiken des Außenhandels mit Drittländern und zur Aufhebung der Verordnung (EG) Nr. 1172/95 des Rates, A6-0267/2008 vom 26. Juni 2010, Berichterstatter: Helmuth Markov; Europäisches Parlament: Bericht des Ausschusses für internationalen Handel über den Vorschlag für eine Verordnung des Europäischen Parlaments und des Rates über Zwangslizenzen für Patente an der Herstellung von Arzneimitteln, die für die Ausfuhr in Länder mit Problemen im Bereich der öffentlichen Gesundheit bestimmt sind, A6-0242/2005 vom 19. Juli 2005, Berichterstatter: Johan Van Hecke.

39 So wurde das Europäische Parlament bei wichtigen Abkommen wie der wirtschaftlichen Partnerschaft zwischen der Europäischen Union und Mexiko oder bei Makrofinanzhilfen für den Libanon, Kosovo oder Georgien lediglich konsultiert. 
ben wir hier einen Akteur, der realen Einfluss hat, der konsultiert und informiert werden muss. Und nicht nur das: Vom ,Ja' und ,Nein' des Europäischen Parlaments hängt beispielsweise ab, ob die Handelsbeziehungen mit Südkorea aufgenommen werden. ${ }^{40}$

Wir haben hier europaweit eine einzigartige Situation: Die Tatsache, dass ein Parlament Entscheidungsrechte im Bereich der Handelspolitik besitzt, die klassisch in die ausschließliche Zuständigkeit der Exekutiven fallen, ist etwas völlig Neues in Europa! Der Deutsche Bundestag hat bisher zum Beispiel nicht annähernd so weitreichenden Einfluss auf die Handelspolitik der Bundesregierung, wie dies jetzt das Europäische Parlament gegenüber der Exekutive erhält. Das gleiche Bild findet sich in den anderen europäischen Ländern. Eine Praxis, die zum Beispiel in den USA schon lange existiert, wird nun zum ersten Mal einem Parlament in Europa zugestanden: dem Europäischen Parlament.

Diese neuen Kompetenzen werden langfristig auch Auswirkungen auf die interne Arbeit des Europäischen Parlaments haben, zum Beispiel im Verhältnis des INTA zu den traditionellen außenpolitischen Ausschüssen wie dem AFET, dem DEVE oder dem Unterausschuss Menschenrechte. So werden mehr informelle Treffen und formale gemeinsame Sitzungen stattfinden müssen, sowohl auf Fraktions- als auch auf Ausschussebene, um sich in wichtigen Fragen, die sowohl die Handelspolitik als auch sicherheits- oder entwicklungspolitische Fragen betreffen, auf eine gemeinsame Linie des Europäischen Parlaments zu verständigen, die dann gemeinsam gegenüber Rat und Kommission vertreten wird. Hier muss das Europäische Parlament ansetzen: Es muss intern eine Kohärenz in außenpolitischen Fragen erarbeiten, um insgesamt auf europäischer Ebene mehr Kohärenz zu erzwingen. Deswegen muss es zuerst intern durch eine bessere gegenseitige Information und Abstimmung seiner außenpolitischen Ausschüsse eine Einheitlichkeit der Positionen schaffen. Denn Kohärenz ist schließlich eines der Leitmotive des Lissabon-Vertrags. Deswegen wurde auch das Amt der Hohen Vertreterin der Union für Außen- und Sicherheitspolitik, die zugleich Vizepräsidentin der Kommission ist, geschaffen und deswegen wird ein Europäischer Auswärtiger Dienst (EAD) eingerichtet. Unter dessen Dach soll die „Gemeinsame Außen- und Sicherheitspolitik und Gemeinsame Sicherheits- und Verteidigungspolitik“ (GASP/GSVP) mit den Generaldirektionen für Entwicklung und Internationale Zusammenarbeit, humanitäre Hilfe und Krisenreaktion der Europäischen Kommission koordiniert werden damit die Europäische Union außenpolitisch als Einheit auftreten kann.

Die Zusammenarbeit der außenpolitischen Ausschüsse des Europäischen Parlaments ist neben gegenseitigen Informations- und Konsultationsmechanismen zudem auf formaler Ebene definiert: So handelt es sich bei dem INTA und dem AFET um sogenannte ,assoziierte Ausschüsse'. Was bedeutet dies konkret? Stellt die Konferenz der Präsidenten, die über die Verteilung von Berichten innerhalb des Europäischen Parlaments an die verschiedenen Ausschüsse entscheidet, fest, dass ein Gegenstand in die Zuständigkeit von zwei oder mehreren Ausschüssen fällt, so wird ein Ausschuss als federführend ernannt, der den Bericht erstellt. Der oder die andere/n Ausschüsse verfassen in diesem Fall eine Stellungnahme zu dem Bericht. Die Zusammenarbeit der Ausschüsse in einem solchen Fall ist in Art. 50 der Geschäftsordnung des Europäischen Parlaments (GO) festgelegt. Hier steht, dass zum Beispiel der Zeitplan von den betroffenen Ausschüssen gemeinsam vereinbart wird und dass der Berichterstatter und der Verfasser der Stellungnahmen ,,sich laufend und gegenseitig [unterrichten] und bemühen [..., ] eine Einigung über die Texte, die sie ihren Ausschüssen vor-

40 Generaldirektion Externe Politikbereiche, Fachbereiche des Europäischen Parlaments: Das Freihandelsabkommen EU-Korea, September 2009, abrufbar unter: http://www.europarl.europa.eu/meetdocs/2009_2014/documents/inta/dv/792/792791/792791de.pdf (letzter Zugriff: 21.06.2010). 
schlagen, und über ihre Haltung zu den Änderungsanträgen zu erzielen. “41 Außerdem kann nach Art. 51 GO die Konferenz der Präsidenten bei einem besonders wichtigen Thema die Anwendung eines Verfahrens mit gemeinsamen Ausschusssitzungen und einer gemeinsamen Abstimmung beschließen.

Die formalen Grundlagen einer engeren Zusammenarbeit des INTA mit den anderen außenpolitischen Ausschüssen ist also gegeben. Wie sich die neuen Kompetenzen des INTA konkret in der Praxis auf die interne Arbeit des Europäischen Parlaments auswirken werden, bleibt noch abzuwarten. Auf jeden Fall wird der Einfluss des Europäischen Parlaments in auswärtigen Fragen über die handelspolitischen Instrumente gestärkt.

\section{Hintergründe: Warum neue Kompetenzen für das Europäische Parlament?}

In Bezug auf die gemeinsame Handelspolitik der Europäischen Union hieß es 2002 im Abschlussbericht der Enquete-Kommission „Globalisierung der Weltwirtschaft“ des Deutschen Bundestages ,Das Demokratiedefizit der Gemeinschaft ist in diesem Bereich [der gemeinsamen Handelspolitik der Europäischen Union] besonders augenfällig. “ ${ }^{42}$ Mit den dargestellten neuen Kompetenzen des Europäischen Parlaments wird nun im Wesentlichen in diesem wichtigen Schlüsselbereich europäischer Politik das kritisierte Demokratiedefizit beseitigt. Die Motivation hierzu muss man auch im allgemeinen Kontext der Stärkung des Europäischen Parlaments durch den Lissabon-Vertrag sehen. Ein Hauptanliegen des Europäischen Konvents war es, die Europäische Union insgesamt demokratischer und transparenter zu gestalten. So wird das Europäische Parlament durch den Lissabon-Vertrag zum gleichberechtigten Mitgesetzgeber in fast allen Bereichen europäischer Politik. Das Mitentscheidungsverfahren wird zum ordentlichen Gesetzgebungsverfahren und dessen Anwendung ausgeweitet. 95 Prozent der EU-Gesetze werden gleichberechtigt vom Europäischen Parlament und dem Ministerrat verabschiedet und die Europäische Union entwickelt sich zu einem ,echten' Zweikammer-System. Die qualifizierten Mehrheitsentscheidungen im Rat werden mit einigen wenigen Ausnahmen zur Regel.

Zudem hat das Europäische Parlament jetzt die vollständigen Haushaltsrechte einschließlich jener über die Agrarausgaben und damit die Mitentscheidung bei allen Haushaltsfragen.

Ebenso unterliegt die Kontrolle der europäischen Exekutive stärker als bisher dem Europäischen Parlament. Es wählt zum Beispiel den Präsidenten der Europäischen Kommission und auch der Hohe Vertreter der Union für Außen- und Sicherheitspolitik/Vizepräsident der Kommission muss durch das Europäische Parlament bestätigt werden. Auch wird die Zustimmung des Europäischen Parlaments bei allen Verträgen mit Drittstaaten verlangt. Die Europäische Union erhält durch den Lissabon-Vertrag eine eigene umfassende Rechtspersönlichkeit und somit die Möglichkeit, Verträge mit Drittstaaten abzuschließen.

Vor diesem Hintergrund des allgemeinen Machtzuwaches des Europäischen Parlaments, war es folgerichtig, dass gerade in dem Schlüsselbereich der GHP, in dem die Europäische Union nun ein deutlich größeres Gewicht zu verzeichnen hat, demokratische Kontrolle und Transparenz erhöht wurden, indem man dem Europäischen Parlament und dem INTA die dementsprechenden neuen Kompetenzen zuschrieb.

41 Europäisches Parlament: Geschäftsordnung des Europäischen Parlaments, 7. Legislaturperiode, Dezember 2009, hier S. 38.

42 Deutscher Bundestag: Schlussbericht der Enquete-Kommission Globalisierung der Weltwirtschaft - Herausforderungen und Antworten, Bundestagsdrucksache 14/9200, S. 158. 


\section{Die Aufwertung des Europäischen Parlaments - Konsequenzen für das Zusammen- spiel der handelspolitischen Akteure}

Ein ,Ja“ oder ,Nein' des Europäischen Parlaments am Ende des Verfahrens ist nicht das Entscheidende, sondern das Drohen mit dem ,Nein'. Hieraus erwächst ihm der Einfluss, das Mandat und die Verhandlungen effektiv zu beeinflussen. Die Rechnung ist insofern relativ einfach: Wer meint, künftig am Parlament vorbei in internationale Verhandlungen treten zu können, handelt grob fahrlässig und auf die Gefahr des politischen Selbstmords hin. Denn wer bei der Mandatserstellung und Ausübung des Mandats nicht die Meinung desjenigen berücksichtigt, der am Ende zustimmen muss, der kann eine bittere Überraschung erleben wie kürzlich im Fall des SWIFT-Abkommens, das ohne ausreichende Konsultierung des Europäischen Parlaments von Kommission und Rat ausgehandelt worden war und deswegen letztendlich an der Ablehnung des Europäischen Parlaments scheiterte. Die Lehren hieraus werden hoffentlich rasch gezogen. Das SWIFT-Abkommen zählt zwar nicht zur Handelspolitik, aber das Verfahren ist ähnlich gelagert. Denn es handelt sich bei dem SWIFT-Abkommen über den Datenaustausch zwischen Europa und den USA im Rahmen der Terrorbekämpfung um ein Drittstaatsabkommen. Nach dem Lissabon-Vertrag muss das Europäische Parlament allen Drittstaatsabkommen zustimmen. Ebenso wie es allen Handelsabkommen neuerdings zustimmen muss.

Allerdings ist die strikte Beachtung des Gewaltenteilungsprinzips unerlässlich. Das heißt, es bedarf eines weitreichenden Einflusses des Parlaments auf das Mandat, einer Kontrolle während der Verhandlungen und der Entscheidung am Schluss. In diesen Phasen internationaler Verhandlungen wie auch im Setzen von gesetzgeberischen Rahmenbedingungen liegen die klassischen parlamentarischen Zuständigkeiten. Wenn sich Abgeordnete allerdings selbst ins Verhandlungsteam setzen - was Sache der Exekutive ist - wird das Parlament zum ,Verbündeten', dem mit großer Wahrscheinlichkeit nachher die Kraft fehlt, ,Nein` zu sagen. Ein solches Verhalten steht der demokratischen Kontrolle und Transparenz entgegen. Durch gemischte Zusammenarbeit zwischen den Organen können deren Funktionen in falsche Hände gelangen, das Parlament liefe dann Gefahr auf Dauer ein ,Gefangener seiner Selbst zu werden, weil es seine Unabhängigkeit preisgegeben hätte.

Inwiefern das neue Zusammenspiel von Europäischem Parlament und Kommission funktioniert, wird sich in Zukunft noch zeigen müssen. Alle Seiten müssen sich an die neue Rolle des Europäischen Parlaments gewöhnen. Das erste Handelsabkommen, das mit Zustimmung des Europäischen Parlaments verabschiedet wird, wird das Abkommen mit Südkorea sein. Dieses Abkommen ist nicht unumstritten. So befürchtet vor allem die Elektro- und Autoindustrie Wettbewerbsnachteile durch das Abkommen. Es fordert eine Nachverhandlung. Das Europäische Parlament kann zwar das Abkommen nicht ändern, da es sich hier um den Bereich der vertraglichen GHP handelt und es hier ein Zustimmungs-, aber kein Mitentscheidungsrecht hat. Die Kommission drängt derweil trotz der fehlenden Ratifizierung durch das Europäische Parlament schon einige Mitgliedstaaten auf die teilweise Inkraftsetzung des Abkommens. Dies darf nicht sein, da so die Zustimmung des Europäische Parlaments zur reinen Formsache degradiert würde. Das Europäische Parlament wird die Tatsachen prüfen und hat auch eine eigene Untersuchung zu den wirtschaftlichen Folgen des Abkommens eingeleitet. Auf Grundlage dieser Maßstäbe wird es dann zu einer Entscheidung kommen. Es lässt sich dabei weder von der Kommission noch von den Mitgliedstaaten, noch von den Interessenvertretern unter Druck setzen. Dies nur als Beispiel, dass in Zukunft die Kommission das Europäische Parlament frühzeitig informieren sollte, sodass es nicht zu unnötigen Verzögerungen bei der Verabschiedung solch wichtiger Abkommen wie dem mit Südkorea kommt. 
Auch die geplanten Handelsabkommen mit Peru und Kolumbien sind umstritten, unter anderem wegen der Menschenrechtssituation vor Ort. Diese liegen aber zurzeit noch bei der Kommission und sind dem Europäischen Parlament noch nicht vorgelegt worden. Eine frühe Abstimmung und Gespräche sind hier notwendig. Die Handelsabkommen mit Kolumbien und Peru könnten also ein erster Prüfstein für das neue Zusammenspiel von Kommission und INTA werden.

Dass sich aber schon erste Konsequenzen aus den neuen Kompetenzen des Europäischen Parlaments ergeben, zeigte die kürzliche Veröffentlichung der Dokumente zur Produktpiraterie (,ACTA“43) auf erheblichen Druck der EU-Parlamentarier auf die Kommission hin. Diese hatte seit 2008 mit den USA, Japan, der Schweiz und Kanada geheime Verhandlungen zur Produktpiraterie geführt. Nachdem dies öffentlich wurde, forderte das Europäische Parlament Zugang zu den Dokumenten. Diesem Druck gab die Kommission am 21. April 2010 nach. Eine erste Überprüfung der Dokumente durch das Europäische Parlament ergab, dass nach seiner Auffassung viele Elemente wie Internetsperren oder Dritthaftungen für Serviceprovider aus dem Abkommensentwurf gestrichen werden sollten. Hier sieht man die Kontrollfunktion, die das Europäische Parlament ausüben kann. Zwar sieht das Europäische Parlament es ähnlich wie die Kommission: Produktpiraterie muss bekämpft und Urheberrechte müssen geschützt werden, aber das darf nicht zu einer Umgehung europäischer Gesetzgebung führen.

Kürzlich hieß es im Handelsblatt: ,US-Lobbyisten putzen bei EU-Abgeordneten Klinken. ${ }^{44}$ Diese Formulierung ist zugegeben reichlich polemisch, aber sie zeigt deutlich, dass auch bei unseren Partnern im Ausland die neuen Kompetenzen des Europäischen Parlaments wahrgenommen werden. In den USA sieht man beispielsweise nun, dass man beim europäischen Partner eine zusätzliche Kraft mit einbeziehen muss und dass in der Handelspolitik Fragen der demokratischen Kontrolle und Legitimation in Zukunft eine bedeutende Rolle spielen werden.

\section{Worauf in Zukunft zu achten ist - einige Empfehlungen für eine Außenpolitik aus ei- nem Guss}

Die Macht, die die Handelspolitik für die Gestaltung der globalen Ordnung hat, strahlt künftig in Verbindung mit den neuen Befugnissen des Parlaments in der Handelspolitik auch auf andere Bereiche aus. Zum einen wird die Europäische Union in Zukunft wohl häufiger mit gemischten Verträgen konfrontiert, in denen nicht nur Handelsfragen, sondern auch andere politische Fragen behandelt werden. Dies hängt auch damit zusammen, dass die Europäische Union jetzt über eine einheitliche Rechtspersönlichkeit verfügt. Die Verzahnung von Außenhandelspolitik und anderen außenpolitischen Bereichen wird besonders bei Handelskonflikten deutlich, zum Beispiel beim Importverbot für Rindfleisch aus den USA und Kanada, das mit Wachstumshormonen behandelt wurde, die in der Europäischen Union verboten sind, oder bei dem seit 1997 bestehenden Importverbot für mit Chlor behandeltem Hühnchenfleisch aus den USA. Hier steht die Europäische Union aktuell unter Druck dieses aufzuheben. ${ }^{45}$ Oftmals sind mit großen handelspolitischen Prozessen sensible Fragen verbunden. Es geht um den Schutz der einheimischen Produzenten, die gegenüber Import-Produzenten benachteiligt werden, und zugleich um den liberalen Handel und damit um die Frage, was wiegt hier mehr?

43 „Anti-Counterfeiting Trade Agreement.“

44 Handelsblatt: US-Lobbyisten denken um: Klinken putzen bei EU-Abgeordneten, 22.04.2010.

45 Vgl. Quisthoudt-Rowohl: Alle Macht in Brüssel, 2009, S. 148. 
Auch in Bezug auf China wird die Überschneidung von Handelspolitik und anderen auBenpolitischen Aspekten deutlich: Wenn die Europäische Union ein Handelsabkommen mit China abschließt, stehen auch Fragen wie die Achtung der Menschenrechte im Raum. Hier muss sich die Europäische Union künftig besser koordinieren. Es darf zum Beispiel nicht sein, dass die Hohe Vertreterin der Union für Außen- und Sicherheitspolitik gerade eine Pressemitteilung veröffentlicht, in der sie die Menschenrechtslage in China kritisiert, und die Europäische Union zugleich ein großes Handelsgeschäft mit China abschließt. Ein anderes Beispiel ist Iran: So steht Europa gegenwärtig vor der vierten Generation von Sanktionen gegenüber dem Iran. Diese betreffen in vielen Bereichen Handelspolitik und Sicherheitspolitik. Entscheidend ist, wie hier das Mischverhältnis austariert wird.

Dieser neue ,Mix ‘ wird in internationalen Verträgen schrittweise Bedeutung erlangen. Es geht dann darum, wie man über die Handelspolitik hinaus auf breiter Linie Politik beeinflussen kann, Menschenrechtsfragen, Fragen, die Umweltschutzstandards oder soziale Rechte betreffen. Das Europäische Parlament muss sich dafür einsetzen, dass dementsprechende Klauseln, die in den Handelsabkommen oder Assoziierungsverträgen integriert sind, umgesetzt werden. Das Europäische Parlament muss also darauf achten, dass diese nicht nur, auf dem Papier stehen', sondern auch angewendet werden. Dieses darf aber keineswegs protektionistischen Zielen dienen, sondern muss in einer ausbalancierten Art und Weise geschehen.

Das Europäische Parlament hat ein Interesse daran, außen- und handelspolitische Ziele miteinander so in Einklang zu bringen, dass die Summe die Europäische Union als Ganzes stärkt. Innerhalb des Parlaments gilt es daher auch zu überlegen, wie die Hauptakteure in den Ausschüssen für Außenpolitik, für Handelspolitik und für Entwicklungspolitik effiziente Methoden der Kooperation und des Meinungsaustauschs entwickeln können, damit die relative Stärke der Europäischen Union für zentrale politische Ziele des Europäischen Parlaments genutzt werden kann. Der Kommissionspräsident hat zu Recht auf Art. 17 EUV hingewiesen, nachdem für alle auswärtigen Beziehungen mit Ausnahme der GASP/GSVP die Kommission zuständig ist. Art. 22 Abs. 2 EUV besagt auch, dass die Kommission Vorschläge in allen Fragen der auswärtigen Beziehungen unterbreiten kann, erneut und eben nur mit Ausnahme der GASP/GSVP. ${ }^{46}$ Dies bedeutet, dass die Handelspolitik wie die ehemaligen Gemeinschaftszuständigkeiten nicht intergouvernemental be- und verhandelt werden. Und auch dieses bedeutet eine große Stärkung für das Parlament und seinen handelspolitischen Ausschuss. Was nicht in die Zuständigkeiten des künftigen EAD fällt, sondern in den bisherigen Zuständigkeiten der Kommission verbleibt, bedarf einer engen, dem Integrationsprinzip folgenden Verbindung durch eine gemeinsame außenpolitische Strategie sowohl auf der exekutiven Ebene als auch im Hinblick auf die Zusammenarbeit zwischen dem Handelsausschuss und dem Auswärtigen Ausschuss. Denn so heißt es in Art. 21 Abs. 3 EUV: „Die Union achtet auf die Kohärenz zwischen den einzelnen Bereichen ihres auswärtigen Handelns sowie zwischen diesen und ihren übrigen Politikbereichen. Der Rat und die Kommission, die vom Hohen Vertreter der Union für Außen- und Sicherheitspolitik unterstützt werden, stellen diese Kohärenz sicher und arbeiten zu diesem Zweck zusammen.“

Wichtig ist deswegen auch, dass Rückkopplungsmechanismen zwischen dem neuen EAD, der Generaldirektion Handel der Kommission und dem Europäischen Parlament etab-

46 Art. 22 Abs. 2 EUV besagt, dass der Hohe Vertreter der Union für Außen- und Sicherheitspolitik und die Kommission dem Rat gemeinsame Vorschläge vorlegen können, ,wobei der Hohe Vertreter für den Bereich der Gemeinsamen Außen- und Sicherheitspolitik und die Kommission für die anderen Bereiche des auswärtigen Handelns zuständig ist.“ 
liert werden. Zurzeit laufen dazu intensive Überlegungen im INTA, wie man die Kohärenz gewährleisten kann. Eine Überlegung ist zum Beispiel, Einheiten der Generaldirektion Handel der Kommission in den EU-Delegationen zu integrieren.

Abschließend lässt sich also feststellen: Der Lissabon-Vertrag stärkt die Rechte des Europäischen Parlaments im Allgemeinen im Sinne von mehr demokratischer Kontrolle, Transparenz und Entscheidungsrechten. Die Außenhandelspolitik bietet dafür ein Musterbeispiel. Dies hat schon kurzfristig zu einem neuen Machtgefüge geführt. Zukünftig muss eine engere Koordination der anderen europäischen Akteure wie Kommission und Rat mit dem Europäischen Parlament stattfinden, wenn man Konflikte vermeiden will. Wenn das Europäische Parlament es schafft, die notwendige Härte zu entwickeln, dann kann sich aus den neuen Rechten und Pflichten des Parlaments eine wirkliche demokratische Legitimation der Handelspolitik entwickeln. Und wenn die Europäische Union es im Allgemeinen schafft, alle Aspekte der auswärtigen Beziehungen miteinander zu verbinden und eine echte ,Außenpolitik aus einem Guss` zu schaffen, dann sollte das Bild vom wirtschaftlichen Riesen und politischen Zwerg endgültig überholt sein.

\section{Legitimität supranationaler Herrschaft und liberaler Nationalismus}

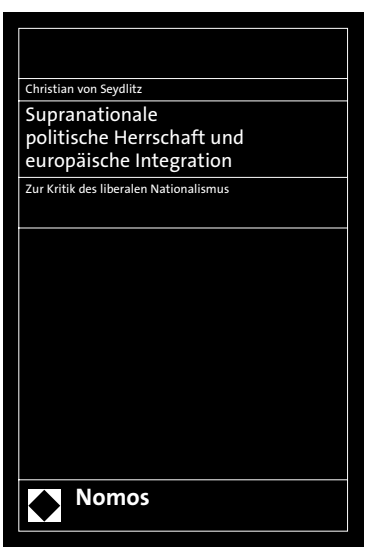

\section{Supranationale politische Herrschaft und europäische Integration}

Zur Kritik des liberalen Nationalismus

Von Christian von Seydlitz

2010, 256 S., brosch., 49,90€, ISBN 978-3-8329-5666-O

Hauptziel der Arbeit ist die Untersuchung der Frage, inwiefern der liberale Nationalismus als normative Theorie supranationaler Herrschaft geeignet ist. Nach der interdisziplinären Analyse der sozialwissenschaftlichen und europarechtlichen Legitimitätsdebatte werden der liberale Nationalismus und seine Verteidigung des Primats des Nationalstaates untersucht.

Bitte bestellen Sie im Buchhandel oder versandkostenfrei unter - www.nomos-shop.de

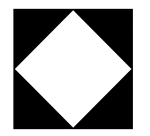

Nomos 